\title{
Dosimetric comparison of volumetric modulated arc therapy with robotic stereotactic radiation therapy in hepatocellular carcinoma
}

\author{
Eun Kyung Paik, MD1, Mi-Sook Kim, MD, PhD¹, Chul Won Choi, MD², Won II Jang, MD', \\ Sung Hyun Lee, MS', Sang Hyoun Choi, PhD¹, Kum Bae Kim, PhD', Dong Han Lee, PhD³ \\ 'Department of Radiation Oncology, Korea Institute of Radiological and Medical Sciences, Seoul; \\ ${ }^{2}$ Department of Radiation Oncology, Dongnam Institute of Radiological and Medical Sciences, Busan; \\ ${ }^{3}$ CyberKnife Center, Korea Institute of Radiological and Medical Sciences, Seoul, Korea
}

Purpose: To compare volumetric modulated arc therapy of RapidArc with robotic stereotactic body radiation therapy (SBRT) of CyberKnife in the planning and delivery of SBRT for hepatocellular carcinoma (HCC) treatment by analyzing dosimetric parameters. Materials and Methods: Two radiation treatment plans were generated for 29 HCC patients, one using Eclipse for the RapidArc plan and the other using Multiplan for the CyberKnife plan. The prescription dose was $60 \mathrm{~Gy}$ in 3 fractions. The dosimetric parameters of planning target volume (PTV) coverage and normal tissue sparing in the RapidArc and the CyberKnife plans were analyzed.

Results: The conformity index was $1.05 \pm 0.02$ for the CyberKnife plan, and $1.13 \pm 0.10$ for the RapidArc plan. The homogeneity index was $1.23 \pm 0.01$ for the CyberKnife plan, and $1.10 \pm 0.03$ for the RapidArc plan. For the normal liver, there were significant differences between the two plans in the low-dose regions of $V_{1}$ and $V_{3}$. The normalized volumes of $V_{60}$ for the normal liver in the RapidArc plan were drastically increased when the mean dose of the PTVs in RapidArc plan is equivalent to the mean dose of the PTVs in the CyberKnife plan.

Conclusion: CyberKnife plans show greater dose conformity, especially in small-sized tumors, while RapidArc plans show good dosimetric distribution of low dose sparing in the normal liver and body.

Keywords: Radiosurgery, Hepatocellular carcinoma, Volumetric modulated arc therapy, Robotic radiosurgery

\section{Introduction}

Hepatocellular carcinoma (HCC) is the fifth most common malignancy and leads the third most common cause of cancerrelated death worldwide [1]. The standard treatment for HCC is surgery, which results in 5-year survival rates of 30\% to $70 \%$. However, only less than $20 \%$ of HCC patients are suitable for surgery. Therefore, several alternative modalities of surgery such as transarterial chemoembolization, percutaneous ethanol injection, radiofrequency ablation, sorafenib, or radiotherapy (RT) have been used [2-7]. Although recent advances in RT techniques including intensity-modulated radiation therapy (IMRT), volumetric modulated arc therapy (VMAT), and stereotactic body radiation therapy (SBRT) have augmented the role of RT in HCC [6-12], in the past, RT had a limited role because of the high risk of liver toxicities such as

Received 7 April 2015, Revised 28 August 2015, Accepted 3 September 2015.

Correspondence: Mi-Sook Kim, MD, PhD, Department of Radiation Oncology, Korea Institute of Radiological and Medical Sciences, 75 Nowon-ro, Nowon-gu, Seoul 01812, Korea. Tel: +82-2-970-1264, Fax: +82-2-970-2412, E-mail: mskim@kirams.re.kr

(c) This is an Open Access article distributed under the terms of the Creative Commons Attribution Non-Commercial License (http://creativecommons.org/ licenses/by-nc/4.0/) which permits unrestricted non-commercial use, distribution, and reproduction in any medium, provided the original work is properly cited.

www.e-roj.org 
radiation-induced liver disease (RILD), which is believed to be an almost fatal complication once it occurs [13-15].

SBRT technique can now be used to precisely deliver radiation to tumors at high doses in a small number of fractions and spare surrounding tissues. The liver is regarded as a paralleled organ, thus the delivery of high radiation dose to selected liver regions is considered safe [12]. To deal with RILD for SBRT administration, several liver constraints were recommended. Schefter et al. [16] reported that the minimum normal liver volume that must be spared from receiving a certain dose is $700 \mathrm{~mL}$ and the maximum dose allowed to this critical volume was estimated to be $15 \mathrm{~Gy}$ in 3 fractions. No RILD or other severe toxicities have been observed according to these constraints. Timmerman [17] showed a maximal critical volume dose of $17.1 \mathrm{~Gy}$ for the critical volume of 700 $\mathrm{mL}$, and the AAPM TG 101 [18] suggested a threshold dose of 19.2 Gy for 3 fractions to the minimum critical volume below $700 \mathrm{~mL}$. However, because these understandings of tolerance were generally from retrospective chart review directly from outcomes or via mathematical modeling, all dose recommendations are associated with some uncertainty and must be approached cautiously.

CyberKnife (Accuray Inc., Sunnyvale, CA, USA) is a nongantry-based frameless robotic system dedicated for stereotactic radiation delivery used world-widely. It has adopted a tracking system to compensate for tumor movement due to breathing. The highly flexible non-isocentric beams deliver highly conformal treatment with steep dose gradients at the target-normal tissue boundary $[19,20]$. However, flexible multibeam direction using robotic arm would increase more integral radiation dose to the body than LINAC-based SBRT implication machines. According to recent concerns of secondary cancer induced by low-dose radiation, the whole body dose differences between the two machines are of special concern. RapidArc (Varian Medical System Inc., Palo Alto, CA, USA) designed for 3D conformal RT or IMRT, is a form of conventional gantry-based LINAC VMAT machine that incorporates variable gantry motion and dose rate, with continuously moving multi-leaf collimators (MLCs) [21]. Although this machine is not dedicated for SBRT, being highly precise with high output of monitor unit (MU) and having shorter planning and delivery time, it could provide an alternative modality to CyberKnife for SBRT implication.

As these two machines have very different mechanical characteristics, they are expected to have both strong and weak points in implication of SBRT and dose distributions. However, the clinical dosimetric comparisons for those modalities are somewhat challenging $[19,22,23]$, and there are limited studies that compare the dosimetric differences for liver cancer patients.

The comparison of dosimetric parameters does not indicate the comparison of clinically effective doses, due to the differences in the systems dealing with clinical tumor motion, and mechanical boundary of accuracy and error for both RapidArc and CyberKnife. CyberKnife is a machine that can deliver doses using respiratory gated technique, while RapidArc delivers doses mainly with an internal target volume (ITV) based technique. Also, differences in the beam delivery system exists between the 2 systems, such as non-coplanar vs. coplanar orientations, MLCs vs. circular collimators, and limited number of fields vs. hundreds of fields, etc. Those differences are beyond the scope of our study and we aim to focus on the planning study to determine the feasibility of using VMAT of RapidArc plan-based SBRT for the liver cancer treatment through comparison of target and normal tissue dosimetric parameters with robotic SBRT system using the CyberKnife plan.

\section{Materials and Methods}

\section{Patient characteristics and image acquisition}

A total of 29 HCC patients who were treated from January 2009 to December 2013 at Korea Cancer Center Hospital, Korea Institute of Radiological and Medical Sciences were enrolled.

Table 1. Characteristics of hepatocellular carcinoma patients who received stereotactic body radiation therapy $(n=29)$

\begin{tabular}{lc}
\hline \multicolumn{1}{c}{ Characteristic } & Value \\
\hline Sex & 18 \\
Male & 11 \\
Female & $66(39-81)$ \\
Age $(\mathrm{yr})$, median (range) & \\
BCLC stage & 10 \\
A & 9 \\
B & 10 \\
C & \\
PTV $\left(\mathrm{cm}^{3}\right)$ & 10 \\
$<50$ & 7 \\
$50-99$ & 8 \\
$100-199$ & 4 \\
$200-260$ & $48.5 \pm 22.3$ \\
Longest diameter of PTV $(\mathrm{mm})$ & $1,050.1 \pm 201.1$ \\
NLV $\left(\mathrm{cm}^{3}\right)$ & $10.7 \pm 8.7$ \\
PTV/NLV $(\%)$ & \\
\hline
\end{tabular}

Values are presented as number or mean \pm standard deviation. $\mathrm{BCLC}$, Barcelona clinic liver cancer; PTV, planning target volume; NLV, normal liver volume. 
All patients were treated with SBRT using RapidArc (40-60 Gy, 3-5 fractions). Patient characteristics are summarized in Table 1.

All simulation computed tomography (CT) scans were carried out by using a GE LightSpeed RT 16-slice CT scanner (General Electric Healthcare, Waukesha, WI, USA) with the patient in the supine position immobilized by an Alpha Cradle (Smithers Medical Products, North Canton, OH, USA). Scan range was from the apex of the lung to the end of the right kidney. Whole liver and both kidneys were included in simulation CT scans. Contrast-enhanced, free-breathing CT scans were acquired with a $2.5-\mathrm{mm}$ thickness with $1 \mathrm{~mm} \times 1 \mathrm{~mm}$ pixel size in 2 different modes, a helical mode and a slow axial mode, at 3 seconds per slice for each patient. The relatively slow CT images included respiratory movement of the target. After image acquisition, the CT images were transferred into the treatment planning system (Eclipse version 10.0, Varian Medical System Inc.) for target volume delineation.

\section{Target delineation}

Target delineation was performed by an experienced radiation oncologist. The gross tumor volume (GTV) was defined as contrast-enhanced tumor volume on CT scans and delineated in both CT image sets of the helical mode and the slow mode. Therefore, the GTV was referred to as an ITV. The clinical tumor volume (CTV) was not used and the planning target volume (PTV) was calculated by adding a 0 to $7 \mathrm{~mm}$ margin to the GTV in all directions. Organs at risk (OARs) such as liver, right/left lung, spinal cord, stomach, heart, right/left kidney, bowel, and esophagus were contoured. The body defined as the CT scan range was automatically contoured when the CT images were imported to the Eclipse treatment planning system.

\section{Treatment planning}

In the Eclipse system, a new plan was generated for this study. The prescribed dose was 60 Gy in 3 fractions for each patient. At least 95\% of the PTV was covered by the prescription dose. For the dose constraints, we adopted the constraint that at least $700 \mathrm{~mL}$ of normal liver (entire liver volume minus cumulative GTV) should not receive a total dose $\geq 17$ Gy in 3 fractions. The detailed dose constraints for other OARs for treatments given in 3 fractions were as follows: spinal cord (maximum <22 Gy, $0.27 \mathrm{~mL}$ or less <18 Gy), duodenum (maximum $<22.2 \mathrm{~Gy}, 5 \mathrm{~mL}$ or less $<15.6 \mathrm{~Gy}, 10 \mathrm{~mL}$ or less $<12.9 \mathrm{~Gy}$ ), stomach (maximum $<30 \mathrm{~Gy}, 5 \mathrm{~mL}$ or less $<22.5$ Gy). The dose constraints were determined according to the protocols used at our institute as described in previously published articles [12,24].

CT images with target volume and OAR delineation contoured using Eclipse treatment planning software were transferred to the MultiPlan system version 4.5 (Accuray Inc.). Then, for each patient, two different treatment plans were produced using Eclipse for the RapidArc plan and MultiPlan for the CyberKnife. The same dose constraints were adopted for both RapidArc and CyberKnife plans.

For the Eclipse, inverse planning was carried out for each patient using $600 \mathrm{MU} / \mathrm{min}, 6 \mathrm{MV}, 3$ full $360^{\circ}$ arcs; 2 counterclockwise and 1 clockwise, with collimator angles of $0^{\circ}, \pm 45^{\circ}$ and couch angles of $0^{\circ}, \pm 15^{\circ}$, respectively. Three arcs were used due to the MU limitation and couch angles of $\pm 15^{\circ}$ were used to make better tumor conformity in the superior/ inferior direction. Each arc had the same isocenter, which is the geometric center of the PTV, and jaw sizes were adjusted fitting a $0.5-\mathrm{cm}$ margin to the PTV.

For the MultiPlan, inverse plans were generated using 1,000 MU/min, $6 \mathrm{MV}$, VSI system version 9.5. The sequential optimization algorithm was used for the generation of treatment plan and the Ray-tracing algorithm was used for dose calculation, which generated a minimum $10 \mathrm{MU}$ and maximum $250 \mathrm{MU}$. As the CyberKnife Iris collimator was recently developed, it has become possible to use it with a fixed type collimator. While treating a patient, using a fixed collimator extends the treatment time, since the collimator needs to be changed. As for Iris, the treatment time does not take much longer, even if multiple collimators were used, as they do not need to be replaced. Also, higher conformity is achieved in the end, as various collimators are used for treating an irregularly-shaped tumor. Therefore, in performing the CyberKnife treatment plan, 4 Iris collimators were used for optimal conformity. The collimator size was selected using 2/3 of the shortest dimension of the PTV.

The treatment time was set as $50 \pm 5$ minutes at the end of the optimization process for a clinically applicable plan. The doses were prescribed at an isodose line (70\%-80\% of the maximum dose) that covered at least 95\% of the PTV, and OARs were spared with the optimization constraints.

\section{Plan comparison and evaluation}

The dose distribution on PTV was evaluated by using parameters such as homogeneity index $(\mathrm{HI})$ and conformity index $(\mathrm{Cl})$. The $\mathrm{HI}$ was defined as the degree of uniformity of dose within the target and was calculated using the formula as follows:

$$
H I=D_{\max } / R_{x} \text { dose. }
$$

The $D_{\max }$ and $R_{x}$ doses are the maximum dose $\left(D_{\max }\right)$ and 

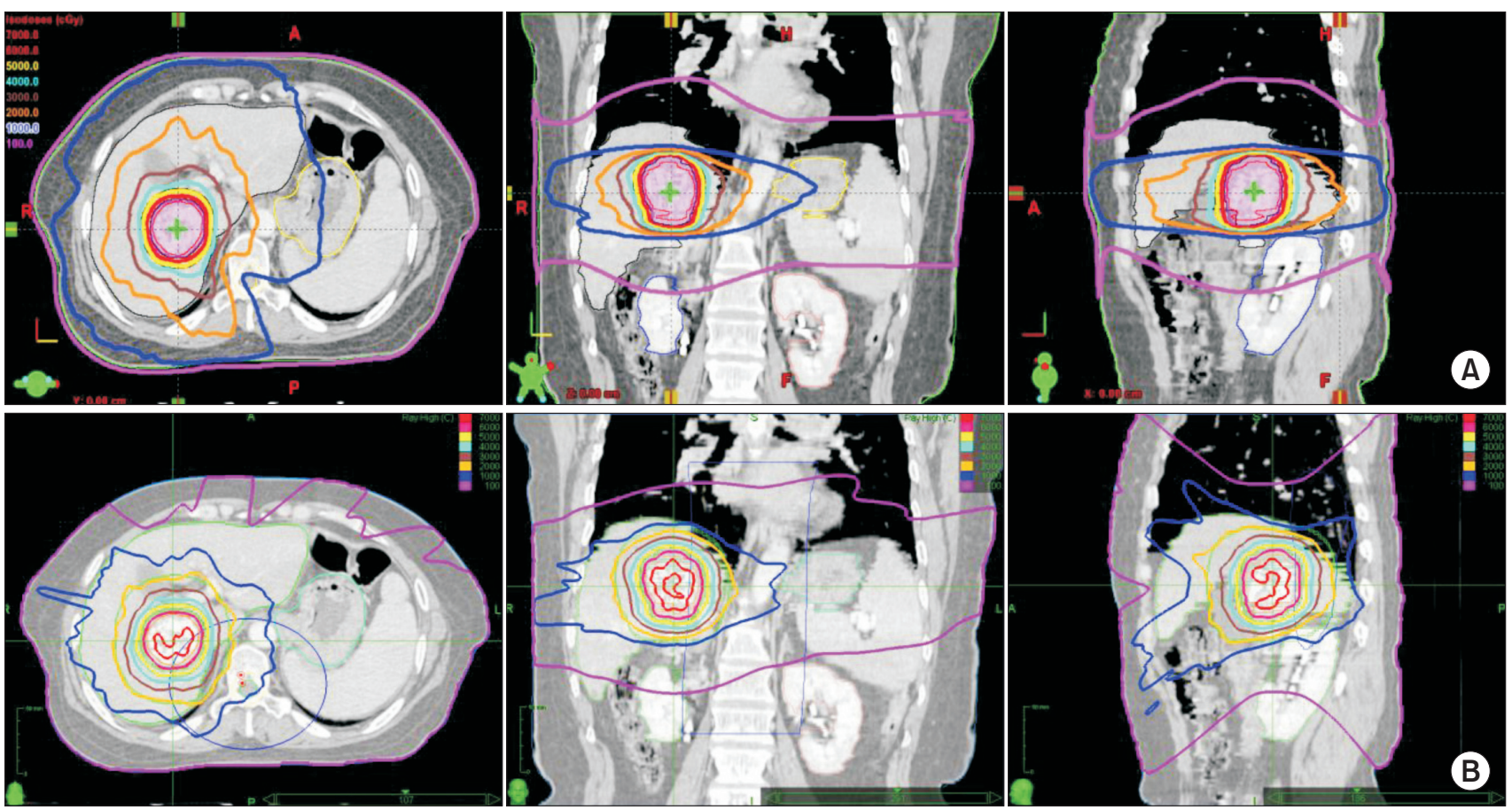

Fig. 1. The axial, coronal, and sagittal views for the (A) RapidArc and (B) CyberKnife plans (red, 70 and 60 Gy; yellow, 50 Gy; cyan, 40 Gy; brown, $30 \mathrm{~Gy}$; orange, $20 \mathrm{~Gy}$; blue, $10 \mathrm{~Gy}$; magenta, $1 \mathrm{~Gy})$.

prescribed dose of the PTV, respectively. The $\mathrm{Cl}$ was described as the ratio between the PTV and the irradiated volume at specified prescription dose and was calculated as follows:

$$
\mathrm{Cl}=\mathrm{PIV} / \mathrm{TV} \text {. }
$$

The prescription isodose volume (PIV) and target volume (TV) was calculated using the volume of the prescription isodose line and the volume of PTV, respectively. For the normal liver and the body, $D_{\text {max }}$ mean dose $\left(D_{\text {mean }}\right), V_{1}, V_{3}, V_{5}, V_{10}, V_{15}, V_{17}$ $V_{20}, V_{30}, V_{40}, V_{50}$, and $V_{60}$ were also evaluated. $V_{\chi}$ represents the relative normal liver volume receiving the dose of $\chi$ Gy or more.

The mean doses for the PTV for both RapidArc and CyberKnife plans were fairy different, especially mean doses in the RapidArc plans were consistently lower than those of the CyberKnife plans. For the identical comparison of target dose, RapidArc plans were individually re-normalized in accordance with mean dose of the PTV in the CyberKnife plan, then, the comparisons in both RapidArc and CyberKnife plan were achieved.

\section{Statistical analysis}

Data are expressed as mean \pm standard deviations. SPSS ver. 14.0 (SPSS Inc., Chicago, IL, USA) software was used for the statistical analyses. The dosimetric differences were analyzed using the Mann-Whitney $U$ test. A p-value less than 0.05 was
Table 2. Dosimetric parameters for planning target volume coverage

\begin{tabular}{lclr}
\hline Variable & RapidArc & CyberKnife & $p$-value \\
\hline$D_{\text {max }}$ (Gy) & $65.8 \pm 1.9$ & $73.5 \pm 0.7$ & $<0.001$ \\
$D_{\text {min }}$ (Gy) & $57.9 \pm 1.4$ & $57.0 \pm 1.9$ & 0.034 \\
$D_{\text {mean }}$ (Gy) & $62.2 \pm 1.0$ & $66.8 \pm 0.6$ & $<0.001$ \\
Cl & $1.13 \pm 0.10$ & $1.05 \pm 0.02$ & $<0.001$ \\
$H I$ & $1.10 \pm 0.03$ & $1.23 \pm 0.01$ & $<0.001$ \\
\hline
\end{tabular}

$\mathrm{D}_{\text {max }}$ maximum dose; $\mathrm{D}_{\text {minı }}$ minimum dose; $\mathrm{D}_{\text {mean }}$ mean dose; $\mathrm{Cl}_{\text {, }}$ conformity index; $\mathrm{HI}$, homogeneity index.

considered statistically significant.

\section{Results}

The isocurve differences between the RapidArc and CyberKnife plans are illustrated in Fig. 1. On the axial view, the low-dose distribution in the RapidArc plan fully covered the whole body contour, while in the CyberKnife plan it covered the body partially. On the coronal and sagittal views, however, the Cyberknife plan showed a wider low-dose distribution than that in the RapidArc plan. Outcomes of dosimetric parameters of the PTV coverage for all patients are summarized in Table 2. Significant differences in $D_{\max }, D_{\text {min }}, D_{\text {mean }}, C l$ and $\mathrm{HI}$ were noted. Fig. 2 shows the differences in $\mathrm{Cl}$ and $\mathrm{HI}$ between the 

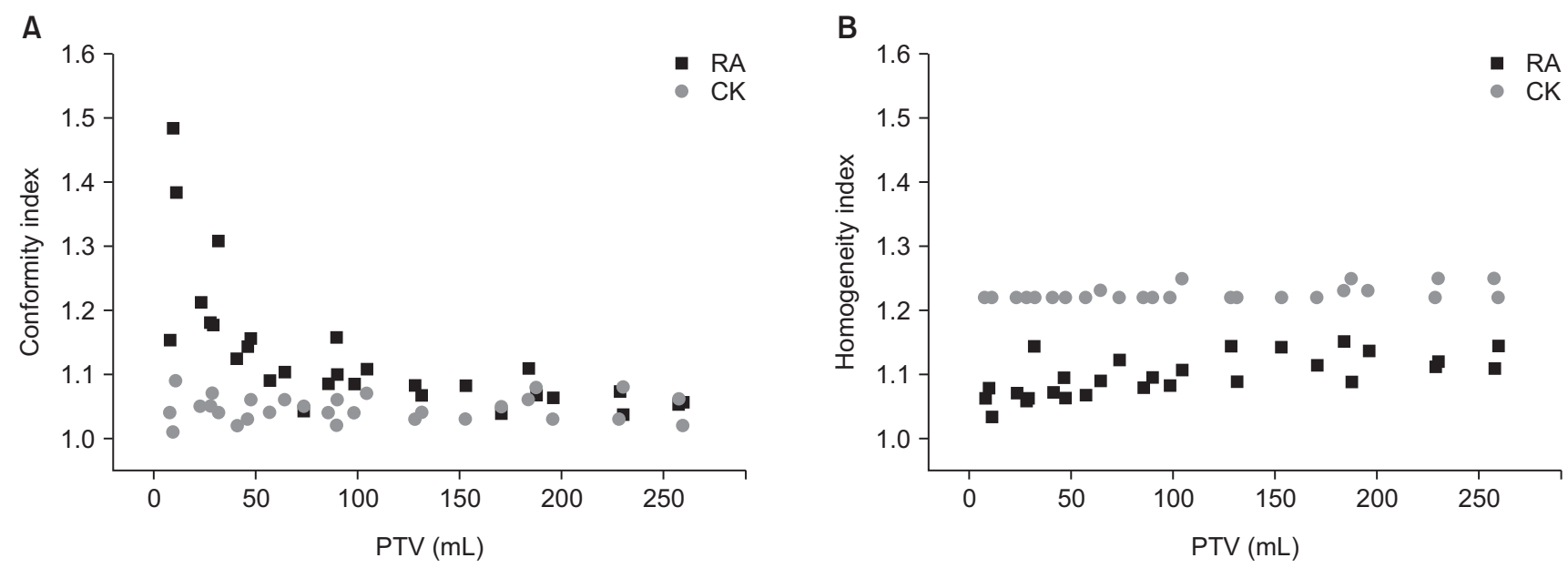

Fig. 2. Comparison of the (A) conformity index and (B) homogeneity index for planning target volume (PTV) in RapidArc (RA) and CyberKnife (CK) plans.

Table 3. Dosimetric parameters for the normal liver

\begin{tabular}{lccc}
\hline$V_{\chi}(\%)$ & RapidArc & CyberKnife & p-value \\
\hline$V_{1}$ & $84.5 \pm 17.3$ & $92.1 \pm 12.4$ & 0.042 \\
$V_{3}$ & $70.7 \pm 21.5$ & $81.5 \pm 18.6$ & 0.036 \\
$V_{5}$ & $64.5 \pm 22.3$ & $71.7 \pm 21.9$ & 0.189 \\
$V_{10}$ & $50.4 \pm 18.7$ & $50.4 \pm 23.3$ & 0.969 \\
$V_{15}$ & $35.9 \pm 15.1$ & $35.1 \pm 19.9$ & 0.703 \\
$V_{17}$ & $30.8 \pm 13.5$ & $30.1 \pm 17.8$ & 0.726 \\
$V_{20}$ & $24.4 \pm 11.3$ & $24.0 \pm 14.6$ & 0.774 \\
$V_{30}$ & $12.1 \pm 6.2$ & $13.5 \pm 8.6$ & 0.680 \\
$V_{40}$ & $6.5 \pm 3.5$ & $8.2 \pm 5.4$ & 0.347 \\
$V_{50}$ & $3.3 \pm 1.7$ & $4.2 \pm 2.8$ & 0.355 \\
$V_{60}$ & $0.4 \pm 0.2$ & $0.3 \pm 0.2$ & 0.011 \\
$D_{\text {max }}$ (Gy) & $64.7 \pm 1.5$ & $64.3 \pm 1.4$ & 0.205 \\
$D_{\text {mean }}$ (Gy) & $13.6 \pm 4.8$ & $14.5 \pm 6.0$ & 0.432 \\
\hline
\end{tabular}

$\mathrm{V}_{\chi 1}$ volume receiving $\chi$ Gy or more; $\mathrm{D}_{\text {max }}$ maximum dose; $\mathrm{D}_{\text {mean }}$ mean dose.

RapidArc plan and the CyberKnife plan. In the CyberKnife plan, all PTV volume range showed conformity better than that of the RapidArc plan. The CyberKnife plan showed a consistent conformity of $1.05 \pm 0.02$ regardless of the PTV volume. In the RapidArc plan conformity was dependent on PTV volume, and the bigger the volume, the lesser the dose conformity. All HIs were consistently high for the CyberKnife plan than that of the RapidArc plan (Fig. 2B).

Table 3 and Fig. 3 show the results of dosimetric parameters for normal liver. We found that there are almost no significant differences between RapidArc and CyberKnife plans except for the low-dose regions of $\mathrm{V}_{1}$ and $\mathrm{V}_{3}$.

Table 4 summarizes the dosimetric parameters for the body. For the body, only $V_{1}$ showed significant differences between

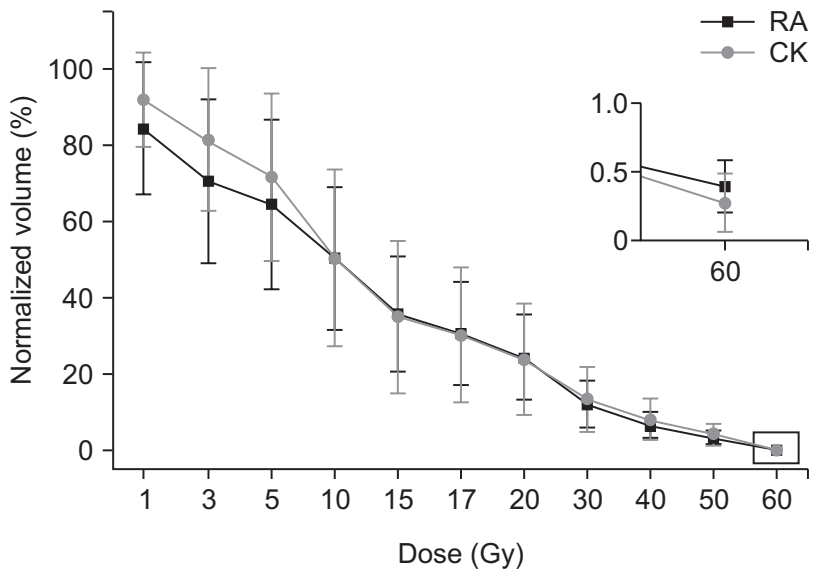

Fig. 3. Averaged normal liver dose-volume histograms of the RapidArc (RA) and CyberKnife (CK) plans.

Table 4. Dosimetric parameters for the body

\begin{tabular}{lccc}
\hline$V_{\chi}(\%)$ & RapidArc & CyberKnife & p-value \\
\hline$V_{1}$ & $34.9 \pm 10.4$ & $61.4 \pm 29.3$ & 0.001 \\
$V_{3}$ & $21.7 \pm 8.1$ & $29.7 \pm 16.7$ & 0.118 \\
$V_{5}$ & $15.8 \pm 7.5$ & $20.0 \pm 12.7$ & 0.308 \\
$V_{10}$ & $8.3 \pm 4.8$ & $9.4 \pm 7.5$ & 0.950 \\
$V_{15}$ & $5.0 \pm 3.3$ & $5.3 \pm 4.6$ & 0.658 \\
$V_{17}$ & $4.2 \pm 2.8$ & $4.2 \pm 3.6$ & 0.617 \\
$V_{20}$ & $3.3 \pm 2.3$ & $3.0 \pm 2.5$ & 0.494 \\
$V_{30}$ & $1.7 \pm 1.3$ & $1.7 \pm 1.4$ & 0.864 \\
$V_{40}$ & $1.1 \pm 0.8$ & $1.2 \pm 1.0$ & 0.785 \\
$V_{50}$ & $0.8 \pm 0.6$ & $0.8 \pm 0.7$ & 0.824 \\
$V_{60}$ & $0.5 \pm 0.4$ & $0.5 \pm 0.4$ & 0.768 \\
$D_{\text {mean }}$ (Gy) & $3.1 \pm 1.5$ & $3.9 \pm 2.3$ & 0.259 \\
\hline
\end{tabular}

$\mathrm{V}_{\chi}$ volume receiving $\chi$ Gy or more; $\mathrm{D}_{\text {mean }}$ mean dose. 

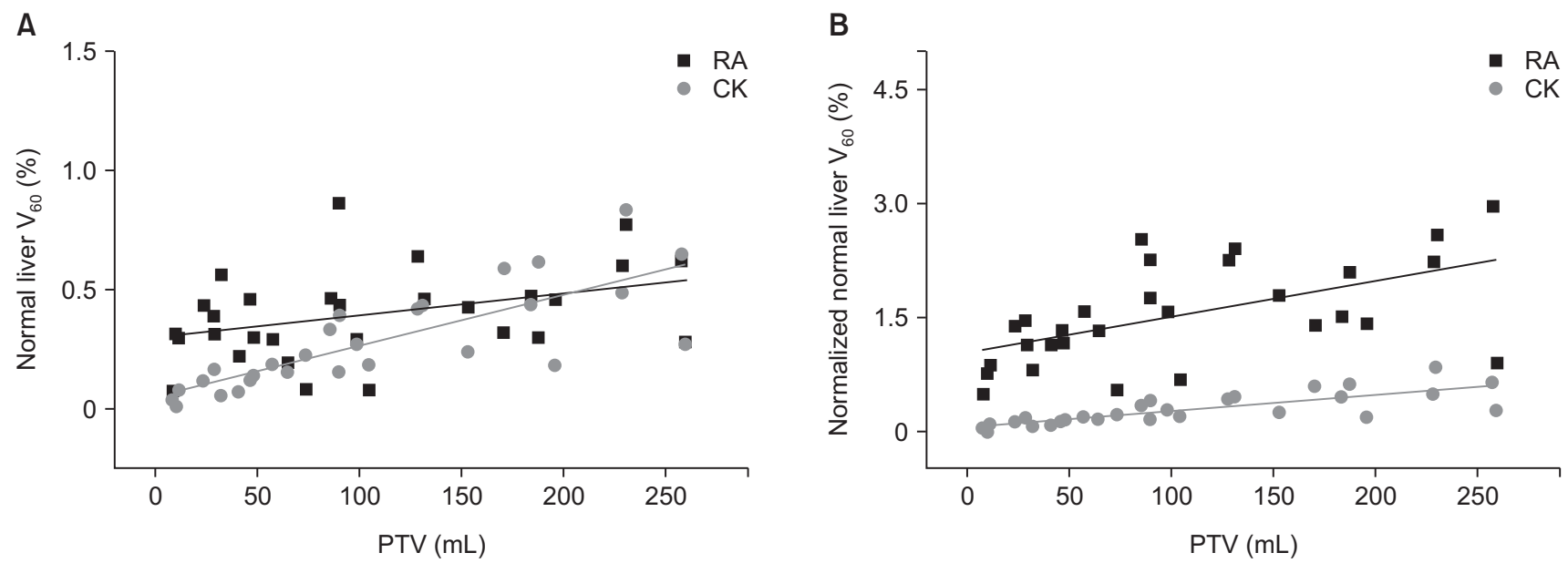

Fig. 4. Comparison of (A) $V_{60}$ of normal liver and (B) normalized $V_{60}$ of normal liver for the RapidArc (RA) and CyberKnife (CK) plans.

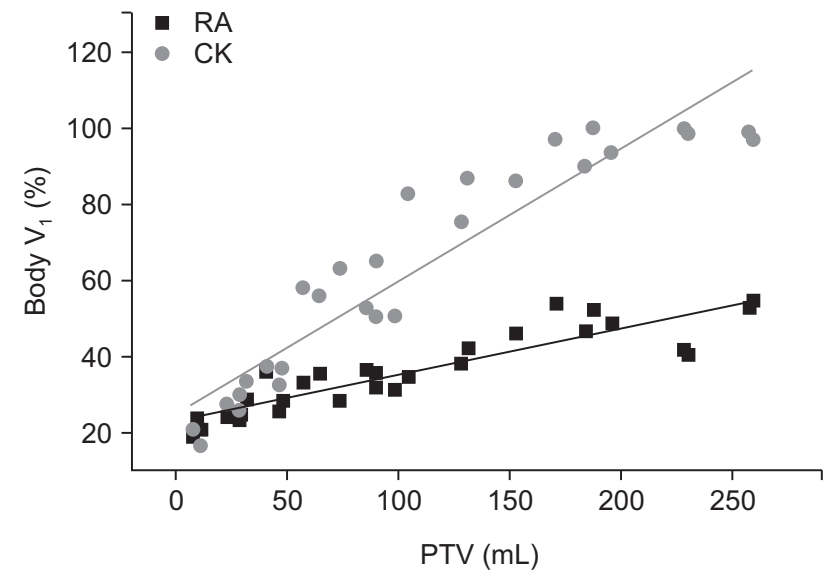

Fig. 5. Comparison of $V_{1}$ of body for the RapidArc (RA) and CyberKnife (CK) plans. PTV, planning target volume.

RapidArc and CyberKnife plans.

Normalized volumes of $V_{60}$ for the normal liver were calculated by setting the mean dose of the PTVs in the RapidArc plans equivalent to the mean dose of the PTVs in the CyberKnife plans. Fig. $4 \mathrm{~A}$ compares the $\mathrm{V}_{60}$ of normal liver between RapidArc and CyberKnife plans and Fig. 4B the normalized $V_{60}$. The normalized $V_{60}$ in RapidArc plans were drastically increased compared to $V_{60}$. Also, normalized volumes of $\mathrm{V}_{60}$ for the normal liver showed gradual increase as the PTV sizes increased.

Fig. 5 shows the differences of $V_{1}$ of the body between the RapidArc and CyberKnife plans. The RapidArc plans consistently achieved a superior sparing of the body in the low-dose region. The difference between the two plans gradually increased as the PTV size increased.

\section{Discussion and Conclusion}

In the past, RT had a limited role in the treatment of HCC patients because of the high risk of liver toxicities such as RILD. However, recent advances in RT techniques including IMRT, VMAT, and SBRT have augmented the role of RT in HCC treatment. Progress in RT technologies could give higher radiation dose to tumor with dose decrement not only in liver but also in other surrounding OARs. There is no study comparing different SBRT techniques in HCC from a dosimetric point of view. Therefore, we investigated the dosimetric differences between SBRT techniques of CyberKnife and RapidArc.

The dosimetric parameters in both CyberKnife and RapidArc plans sufficiently meet the SBRT treatment requirements. For the clinically meaningful region of the normal liver, $V_{15}(35.9 \%$ vs. $35.1 \% ; p=0.703)$ and $V_{17}(30.8 \%$ vs. $30.1 \% ; p=0.726)$ of the RapidArc and CyberKnife plans have shown that there were no significant differences between both modalities under the clinically acceptable levels. But we have seen some discernible differences for the high- and low-dose regions in normal liver.

We observed the distinct dosimetric advantage of the CyberKnife plan over the RapidArc plan at high-dose regions, excellent PTV conformity and normal liver sparing adjacent to PTV. In this study, the $D_{\max }$ and $\mathrm{HI}$ of PTV were higher in the CyberKnife plan than in the RapidArc plan. In other words, RapidArc has an advantage in achieving homogeneous dose distributions in the tumor region. However, homogeneous dose distribution in the tumor region should not be simply considered as an advantage for SBRT. CTV for 3D conformal RT or IMRT usually cover normal tissue surrounding the tumor, 
including microscopic expansion of tumor cells. In contrast, CTV in SBRT usually includes the only the tumor itself. This practice allows dose heterogeneity within the target. Hot spots within the TVs are generally viewed to be clinically acceptable, as long as there is no spillage into normal tissue. It has been hypothesized that hot spots within the central region of a tumor might offer a special advantage in eradicating radio resistant hypoxic cells that might more likely be located there [19]. In liver SBRT, PTV usually includes the tumor itself and limited normal liver. Previously, a clear dose-response relationship in HCC treated by SBRT was reported $[10,12,24]$. Therefore, even though some parts of PTV may receive higher radiation doses than the prescribed dose, higher radiation dose delivery in HCC patients would be necessary to achieve high $D_{\text {max }}$ or $D_{\text {mean }}$ inside the tumor, and thus achieve a better local control rate.

The $V_{60}$ of high-dose region in normal liver were higher in the RapidArc plan than in the CyberKnife plan. This can be explained by the $\mathrm{Cl}$ parameter that RapidArc plan had lower conformity than CyberKnife plan, which means the radiation dose that should be delivered only to the treatment target leaked into the normal liver more in the RapidArc plan than in the CyberKnife plan. In contrast, SBRT studies of prostate cancer case demonstrated higher dose conformity in the RapidArc plan than in the CyberKnife plan [20,25]. But we have shown that the CyberKnife plan consistently achieved less $\mathrm{Cl}$ than the RapidArc plan, especially in small-sized tumors less than $100 \mathrm{~mL}$ (Fig. 2). A Cl greater than 1 indicates that the irradiated volume is greater than the TV and includes healthy tissues [26]. Therefore, in order to minimize the normal tissue toxicity, conformation of high doses to the target and rapid fall-off doses away from the target is critical [19]. As the tumor size gets smaller, it is assumed that RapidArc plan has less conformity and more dose leakage to the normal tissue due to the thickness of MLC which has a limitation in making a corresponding shape when the tumor volume is small, while in CyberKnife, which has a cone collimator system (radius range from 5 to $60 \mathrm{~mm}$ ), showed excellent dose conformity regardless of tumor size.

For the low-dose region of normal liver and body, on the other hand, we observed a distinct dosimetric advantage of the RapidArc plan over the CyberKnife plan. This is well consistent with the results for the prostate SBRT cases indicating that mean low-dose regions of OAR were larger in the CyberKnife plan than in the RapidArc plan $[20,25]$. In this study, the normal liver volume below $10 \mathrm{~Gy}$ in the CyberKnife plan was larger than that of the RapidArc plan, while no significant differences were shown in the dose regions from 10 to 50 Gy. Also, it is reported that fatal hepatic toxicities might be induced at doses lower than the therapeutic dose, which is not effective for tumor control $[27,28]$. Yamashita et al. [29] reported that the incidence of lung toxicity will become higher if large amount of low dose radiation is delivered. Also, Shueng et al. [30] suggested that the $V_{5}$ of the normal lung of only $20 \%$ still potentially induced radiation pneumonitis. In the low dose region of the body, RapidArc plan showed lower dosevolume histogram than CyberKnife plan in $V_{1}$ (34.9 vs. 61.4; $p<0.001)$. Especially, those differences in $V_{1}$ were prominent as the tumor size increased (Fig. 5). It is assumed that as the tumor size increases, the number of non-coplanar radiation beams in CyberKnife increases and this may occur as broadly spread wide low dose regions in the CyberKnife plan compared with the RapidArc plan. The potential risk caused by wide low dose irradiation to the normal liver and body is quite unclear but it should not be neglected that CyberKnife would bring wider low dose irradiation than RapidArc.

Selection of patients for a particular SBRT method is an important issue. However, an important disadvantage of Cyberknife treatment compared to RapidArc treatment was longer delivery time. Treatment time is significantly shorter for LINAC plans (20-30 minutes) compared to CyberKnife plans (about 50 minutes). Longer delivery time could cause patient's discomfort and intra-fractional positioning error, requiring sufficient margin for the adequate coverage of the TV. Therefore, shorter treatment time is of advantage. However, it is known to be able to reduce the treatment margin in the Cyberknife treatment due to a more accurate correction for respiratory motion, and direct comparison of the treatment time between the two plans may not be suitable. In this study, the treatment time of the CyberKnife plan was set as $50 \pm 5$ minutes at the end of the optimization process for a clinically applicable plan. Based on clinical experience, if the treatment time exceeds 40 to 50 minutes, patients have a hard time keeping the same position. This will affect the accuracy and reproducibility of the treatment. However if the optimized Cyberknife treatment time is less than 50 minutes, dosimetric parameters sharply take a turn for the worse. Therefore, the treatment time of the CyberKnife plan was optimized at about 50 minutes.

Our study has some limitations. First, the sample size was small with 29 patients and may not be applicable for all cases. Second, we calculated RapidArc plans using three arcs, a technique not always used in actual treatment planning. These limitations could cause some errors in the interpretation of 
the results. Third, the differences in the beam delivery system between RapidArc and CyberKnife, such as non-coplanar versus coplanar orientations, MLCs versus circular collimators, and limited number of fields versus hundreds of fields, were not evaluated.

In summary, CyberKnife plans showed greater dose conformity, meaning less dose leakage to the normal tissue, thus would increase the dose to the PTV that might improve tumor control probability. Especially, it was evident from the above characteristics in small-sized tumors. However, the advantages of RapidArc, good dosimetric distribution of low dose sparing in normal liver and body and shorter delivery time, showed the possible usage of the SBRT technique using RapidArc in liver cancer patients. Nonetheless, further study is needed to define the effects and to determine the indications for CyberKnife treatment.

\section{Conflict of Interest}

No potential conflict of interest relevant to this article was reported.

\section{References}

1. Bosch FX, Ribes J, Diaz M, Cleries R. Primary liver cancer: worldwide incidence and trends. Gastroenterology 2004;127(5 Suppl 1):S5-S16.

2. Camma C, Schepis F, Orlando A, et al. Transarterial chemoembolization for unresectable hepatocellular carcinoma: metaanalysis of randomized controlled trials. Radiology 2002;224: 47-54.

3. Cheng $A L$, Kang $Y K$, Chen $Z$, et al. Efficacy and safety of sorafenib in patients in the Asia-Pacific region with advanced hepatocellular carcinoma: a phase III randomised, doubleblind, placebo-controlled trial. Lancet Oncol 2009;10:25-34.

4. Shiina $S$, Tateishi $R$, Imamura $M$, et al. Percutaneous ethanol injection for hepatocellular carcinoma: 20-year outcome and prognostic factors. Liver Int 2012;32:1434-42.

5. Tateishi R, Shiina S, Teratani T, et al. Percutaneous radiofrequency ablation for hepatocellular carcinoma: an analysis of 1000 cases. Cancer 2005;103:1201-9.

6. Salhab M, Canelo R. An overview of evidence-based management of hepatocellular carcinoma: a meta-analysis. J Cancer Res Ther 2011;7:463-75.

7. Feng M, Ben-Josef E. Radiation therapy for hepatocellular carcinoma. Semin Radiat Oncol 2011;21:271-7.

8. Kim SH, Kang MK, Yea JW, Kim SK, Choi JH, Oh SA. The impact of beam angle configuration of intensity-modulated radiotherapy in the hepatocellular carcinoma. Radiat Oncol J 2012;30:146-51.

9. Kim YI, Park HC, Lim do H, et al. Changes of the liver volume and the Child-Pugh score after high dose hypofractionated radiotherapy in patients with small hepatocellular carcinoma. Radiat Oncol J 2012;30:189-96.

10. Seo YS, Kim MS, Yoo SY, et al. Preliminary result of stereotactic body radiotherapy as a local salvage treatment for inoperable hepatocellular carcinoma. J Surg Oncol 2010;102:209-14.

11. Shin YJ, Kim MS, Yoo SY, et al. Pilot study of stereotactic body radiotherapy for huge hepatocellular carcinoma unsuitable for other therapies. Tumori 2010;96:65-70.

12. Kang JK, Kim MS, Cho CK, et al. Stereotactic body radiation therapy for inoperable hepatocellular carcinoma as a local salvage treatment after incomplete transarterial chemoembolization. Cancer 2012;118:5424-31.

13. Park W, Lim DH, Paik SW, et al. Local radiotherapy for patients with unresectable hepatocellular carcinoma. Int J Radiat Oncol Biol Phys 2005;61:1143-50.

14. Lawrence TS, Ten Haken RK, Kessler ML, et al. The use of 3-D dose volume analysis to predict radiation hepatitis. Int J Radiat Oncol Biol Phys 1992;23:781-8.

15. Cheng JC, Wu JK, Huang CM, et al. Radiation-induced liver disease after three-dimensional conformal radiotherapy for patients with hepatocellular carcinoma: dosimetric analysis and implication. Int J Radiat Oncol Biol Phys 2002;54:156-62.

16. Schefter TE, Kavanagh BD, Timmerman RD, Cardenes HR, Baron A, Gaspar LE. A phase I trial of stereotactic body radiation therapy (SBRT) for liver metastases. Int J Radiat Oncol Biol Phys 2005;62:1371-8.

17. Timmerman RD. An overview of hypofractionation and introduction to this issue of seminars in radiation oncology. Semin Radiat Oncol 2008;18:215-22.

18. Benedict SH, Yenice KM, Followill D, et al. Stereotactic body radiation therapy: the report of AAPM Task Group 101. Med Phys 2010;37:4078-101.

19. Macdougall ND, Dean C, Muirhead R. Stereotactic body radiotherapy in prostate cancer: is rapidarc a better solution than cyberknife? Clin Oncol (R Coll Radiol) 2014;26:4-9.

20. Wu VW, Tam KW, Tong SM. Evaluation of the influence of tumor location and size on the difference of dose calculation between Ray Tracing algorithm and Fast Monte Carlo algorithm in stereotactic body radiotherapy of non-small cell lung cancer using CyberKnife. J Appl Clin Med Phys 2013;14:68-78.

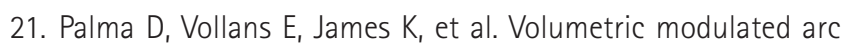
therapy for delivery of prostate radiotherapy: comparison with intensity-modulated radiotherapy and three-dimensional conformal radiotherapy. Int J Radiat Oncol Biol Phys 2008;72: 996-1001.

22. Dutta D, Balaji Subramanian S, Murli V, Sudahar H, Gopalakrishna 
Kurup PG, Potharaju M. Dosimetric comparison of Linac-based (BrainLAB ${ }^{\circledR}$ ) and robotic radiosurgery (CyberKnife ${ }^{\circledR}$ ) stereotactic system plans for acoustic schwannoma. J Neurooncol 2012;106: 637-42.

23. Atalar B, Aydin G, Gungor G, Caglar H, Yapici B, Ozyar E. Dosimetric comparison of robotic and conventional linacbased stereotactic lung irradiation in early-stage lung cancer. Technol Cancer Res Treat 2012;11:249-55.

24. Jang WI, Kim MS, Bae SH, et al. High-dose stereotactic body radiotherapy correlates increased local control and overall survival in patients with inoperable hepatocellular carcinoma. Radiat Oncol 2013;8:250.

25. Lin YW, Lin KH, Ho HW, et al. Treatment plan comparison between stereotactic body radiation therapy techniques for prostate cancer: non-isocentric CyberKnife versus isocentric RapidArc. Phys Med 2014;30:654-61.
26. Feuvret L, Noel G, Mazeron JJ, Bey P. Conformity index: a review. Int J Radiat Oncol Biol Phys 2006;64:333-42.

27. Cochrane AM, Murray-Lyon IM, Brinkley DM, Williams R. Quadruple chemotherapy versus radiotherapy in treatment of primary hepatocellular carcinoma. Cancer 1977;40:609-14.

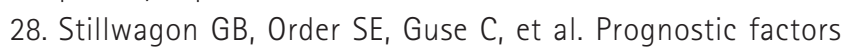
in unresectable hepatocellular cancer: Radiation Therapy Oncology Group Study 83-01. Int J Radiat Oncol Biol Phys 1991;20:65-71.

29. Yamashita $H$, Nakagawa $K$, Nakamura $N$, et al. Exceptionally high incidence of symptomatic grade 2-5 radiation pneumonitis after stereotactic radiation therapy for lung tumors. Radiat Oncol 2007;2:21.

30. Shueng PW, Lin SC, Chang HT, et al. Toxicity risk of non-target organs at risk receiving low-dose radiation: case report. Radiat Oncol 2009;4:71. 